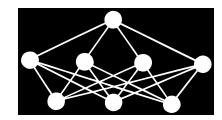

\title{
EDITORIAL
}

\section{PROF. ING. MIRKO NOVÁK, DRSC. PASSED AWAY}

\author{
P. Bouchner*,
}

DOI: $10.14311 /$ NNW.2020.30.006

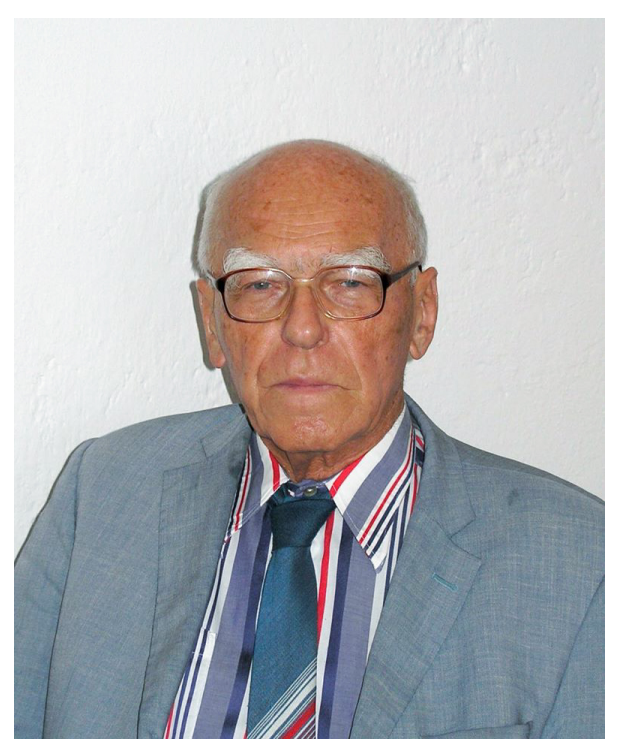

On March $10^{\text {th }}, 2020$ an outstanding man, scientist, teacher visionary, creator, friend and founder of Neural Network World, prof. Ing. Mirko Novák, DrSc. departed from our lives.

Prof. Ing. Mirko Novak, DrSc. was born on September 29, 1930 in Prague, Czechoslovakia. He graduated from Czech University Technical University, Prague in 1952, where he was an assistant professor in the period 1952-1955. In 1956 he joined the Institute of Radioengineering and Electronics of the Czechoslovak Academy of Sciences in Prague, where he was the head of the Department of System Theory. In 1965 and 1966 he was the visiting professor of the Department of Electrical Engineering of New York University. In 1975 he founded a new Institute of Computer Science of the Czechoslovak Academy of Sciences. He was in the position of the director of this Institute for almost 15 years. Since 1965 he was the

\footnotetext{
${ }^{*}$ Petr Bouchner; Department of vehicles, Faculty of transportation Sciences, Czech Technical University of Prague, Czech Republic, E-mail: bouchner@fd.cvut.cz
} 
senior member of the Institute of Electrical and Electronic Engineers, Inc. and in 1988 he became the Corresponding member of the Czechoslovak Academy of Sciences. His research interest in the field of neural networks is mainly in the theory of sensitivity and tolerances of neural networks and of their applications for signal processing, time series prediction and system reliability improvement. He was also interested in internal information systems of living bodies and cells. Prof. Dr. Mirko Novák wrote more than 300 research reports and papers, presented about 200 contributions on scientific conferences, colloquia and seminars and published almost 25 scientific books in Czech, English and Russian, total - more than 500 scientific presentations. At the end of 1994 he was one of the founders of the Joint Laboratory of System Reliability between the Czech Technical University, Prague, Faculty of Transportation Sciences and the Institute of Computer Science of the Academy of Sciences, the Czech Republic. He was a member of the editorial boards of several international scientific journals. Since 1990, he was the editor-in-chief of the international scientific journal Neural Network World for 23 years and a member of the international scientific councils of two foreign scientific magazines. In 1999 he joined the activity of the workgroup for Neuroinformatics of the Global Science Forum OECD and took part in the preparation of the world research program PIN in neuroinformatics. He was the chairman of the Czech National Node for Neuroinformatics and the Czech representative in INCF (International Neuroinformatic Coordination Facility) of GSF OECD.

During this period, he lectured for a long time at several universities - Czech Technical University in Prague, Faculty of Electrical Engineering, Czech University Technical University in Prague, Faculty of Nuclear Sciences and Physical Engineering, University of West Bohemia, New York University, Czech Technical University in Prague, Faculty of Transportation Sciences. Faculty of Transportation Sciences was the one which he helped to start up, where he established a background for bachelor and master courses and led tens of diploma and doctoral theses in engineering informatics and to which he devoted the last 25 years of his life.

It is almost impossible to summarize all important and notable activities and not to forget or prioritize one over the other. Everyone who knew him would surely find his own way, but - at this place - we might remember him as the founder and for many years the editor-in-chief of this journal. At this place an enumeration of merits should be usually presented. But for me it is his spirit penetrating in all aspects of this journal. And to recall it best is to remind his own words which Neural Network World journal was born with. In the following paragraph one can again remind his message which today is as valid as it was almost 30 years ago. Of course, some sketched approaches have been changed during the decades of the editor work as it was necessary to reflect changes in readers' expectations and development of the internet and social media (i.e. book reviews, event invitations and products reviews have already found their place in more flexible and appropriate online platforms), but we kept the text untouched not to damage its authenticity.

Petr Bouchner

editor-in-chief on behalf of steering committee, editorial board and editing office 


\section{THE INAUGURATION OF A NEW JOURNAL ${ }^{1}$}

We welcome you to the first issue of Neural Network World, the first scientific journal published in Middle and Eastern Europe devoted to the problems of neural and mass-parallel computing and information systems. The field of neurocomputing, which roughly speaking involves a good part of this area, is in recent years the one having the steepest increase of interest around the world. Though several wellknown journals dedicated to neural networks and neurocomputers already existed for a few years (and several more appeared last year), none of them comes from this part of the world, where a considerably large interest of many people in this area has a good history. Due to the political changes in our country and thanks the support of IDG Company, Czechoslovakia in Prague, we were able to prepare in very short time the foundation of the journal, in which we hope to create a scientific forum for the free exchange of ideas, knowledge and meanings of all the people interested in neuroscience and in related scientific areas. We hope that in addition to the people living in this part of the world our colleagues from other geographical areas will also contribute to Neural Network World.

The field of neuroscience is very wide. We can take it as dramatic and dynamic scene, illuminated by many beams emitted from different sources, in which the knowledge and experience of various areas comes together. There is a great variety of these sources and it is hard to identify all of them. However, definitely included among we find neurophysiology, mathematics, physics, informatics, cybernetics and electronics (see Fig. 1). Of course, beside these, there are other sources, such as microbiology, molecular genetics on one side, psychology and linguistics on

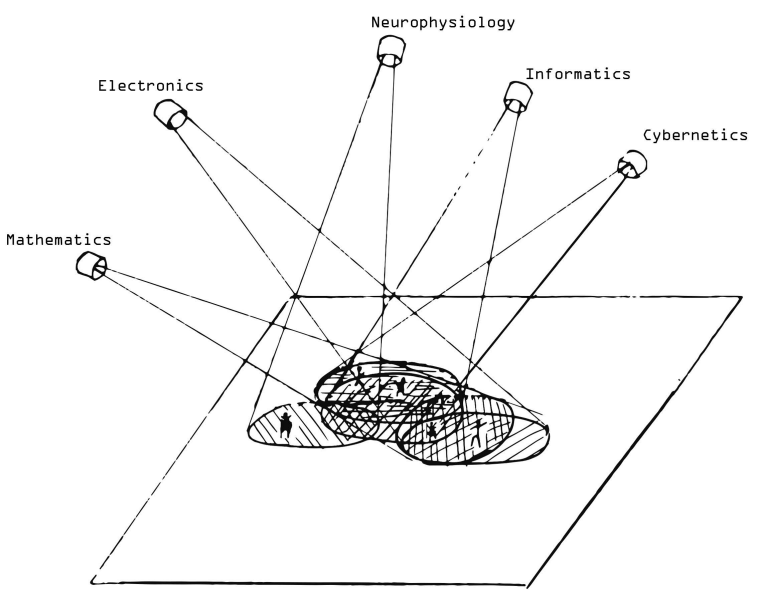

Fig. 1 The field of neuroscience.

the other side and chemistry, communication engineering and various engineering applications intersecting with the beams illuminating the scene, where the authors - the neuroscientists - have to play much more complicated roles. The beams have different colors as, the languages in which the knowledge from individual sources is

\footnotetext{
${ }^{1}$ Neural Network World, Volume 1, Number 1, IDG Co., Czechoslovakia, 1991.
} 


\section{Neural Network World 2/2020}

emitted are not identical. There are also not all of point nature. Some of the, like the source of mathematics e.g., consists of several grouped partial sources. Some have a diffusion character. Therefore, it is very difficult to define their intersection on the scene and to identify the actual color of the particular place, where one or the other actor is acting.

Moreover, the whole scene is not static - it changes very dynamically. The sources emit their beams of knowledge in different parts of the scene, changes their intensity in time (sometimes also stop), switch positions (comes closer together of take distance) and also the actors do not play all their roles by staying in the same place in the scene. The whole scene of neuroscience can therefore be considered by an independent observer as a "Big Chaos". This probably happens often especially to the observer who are close to the bright and dazzling sources of knowledge illuminating the scene.

Nevertheless, there are some positions from which at least some parts of the scene can be observed with satisfactory probability to recognize the sense of the play and to understand their internal relations and laws. I personally belong to the people who believe that the whole is not a large chaotic motion of the Brownian character, but that this a very dramatic and complicated development of the deep and old tendency of the human being to try to understand at least a little bit of its own consciousness and nature.

What role can be played here by the scientific journals specialized more or less to this field? Last fall, after a considerably successful International Symposium of Neural Networks and Neurocomputing NEURONET '90 held in Prague and having been asked by Vladimír Tichý, the manager of IDG Company, Czechoslovakia and to whom I am very grateful for this idea and support in their development, to prepare the publication of a new scientific journal devoted to this field. I needed to try, at least for myself, to answer this question. I came to the feeling that for such a dramatic scene like the one demonstrated schematically in Fig. 1, such journals can represent the screens or mirrors on which not only the interested observers but also the actors can see some parts of the drama in more detail and fixed time.

Therefore, by the use of such tools, the actors - the number of whom is now already high enough - and also the observers can take advantage of having more information about the activity in the distant parts of the scene, which are from their own standpoint not quite clearly visible (see Fig. 2).

Of course, there are now at our disposal several kinds of such screens. Above all, we have here the group of well - known high level scientific journals. By the end of 1990, about 6 of them were known to me. These are:

- Neural Networks, by Pergamon Press,

- International Journal of Neural Systems, by World Scientific,

- IEEE Transactions on Neural Networks, by IEEE,

- Neural Computing, by MIT Press,

- Neural Technology Update, by Elsevier and

- International Journal on Neural Networks. 


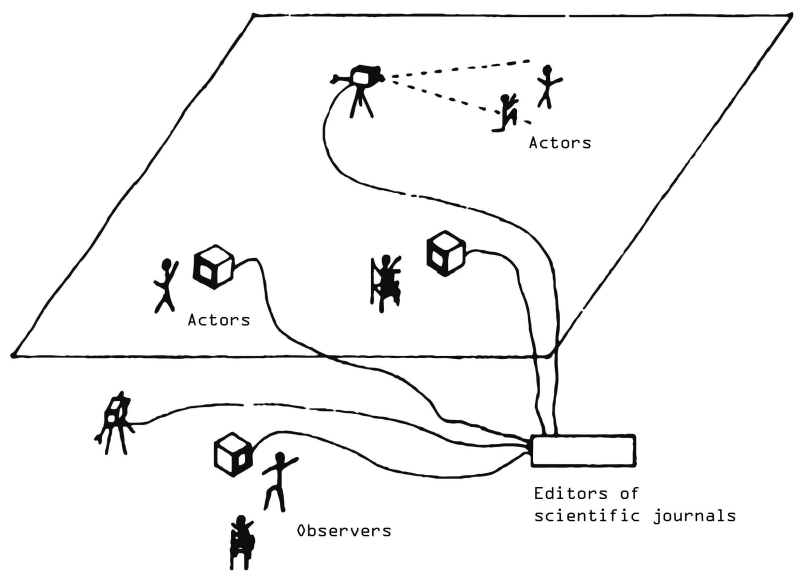

Fig. 2 To the role of scientific journals.

Of course, this list is not complete and I am also sure that in 1991 more such journals will appear. Beside in these, the papers concerning neuroscience problems appear almost regularly in many other scientific journals, like e.g. in the

- IEEE Transactions on Systems, Man and Cybernetics,

- IEEE Transactions on Pattern Analysis and Machine Intelligence,

- IEEE Transactions on Circuits and Systems,

- IEEE Transactions on Information Theory,

- IEEE Control System Magazine,

- IEEE Circuit and Device Magazine,

- IEEE ASSP Magazine,

- Proceedings of the IEEE,

- Computer,

- Spectrum,

- Journal on Cognitive Neuroscience,

- Journal of New Generation Computer Systems,

- Communications of the ACM,

- Biological Cybernetics,

- Expert Systems,

- Simulation, 


\section{Neural Network World 2/2020}

- The American Physical Society,

- Applied Intelligence,

- Applied Optics,

- Optical Letters,

- Physica,

- Nature, etc.

All of these periodicals have their own profile and contribute to the information of both the actors and observers of the neuroscience scene.

Therefore, the second question which we need to answer is: "Is there really space (and if there is, where?) for a new scientific journal in this area?"

The answer to this question is much more difficult. It can be influenced above all by the fact that at present, the total publication capacity of all these periodicals is still evidently much smaller than corresponds to the quickly increasing number of people active in it and having results worth publishing (even if one considers the necessary amount of rejected or long corrected papers). The present-day reality is that many authors have to wait many months for publication and that some results lose therefore a part of their timeliness. The second aspect influencing the answer comes from the regional distribution of the existing journals. Up to now all of them are published in the western hemisphere. However, there are also other parts of the world, where the political barriers are fortunately now disappearing, where there are many people who are interested in this field who can contribute to its development.

Being here in Prague in the heart of Europe just between these two approaching parts of the world, we would like to offer to all of these people the better possibility to publish their ideas and research results and we hope that they will make use of it. We hope also, that such a screening of activity in neuroscience in the East will be interesting for the people from the West and vice versa, so that our western colleagues will present in Neural Network World some of their results which should be relayed here quicker.

Summarizing these and some further aspects, we do hope that we can find our right place between all the other screens and mirrors on the scene.

\section{The scope of Neural Network World}

Nevertheless, the scene of neuroscience is not a flat plane. It can be better compared to the relief of the landscape with many hills and valleys. There is almost impossible to mirror such a complicated object in one screen. Therefore, one needs in any case to concentrate one's interest on some parts of the scene. The question is, how to choose them, how to place the cameras and ho to display the pictures on the screen. Evidently, if the focus will be too narrow, the consciousness of the context can be lost.

We expect that the reasonable compromise between too wide and a too narrow scope of our Journal will be in focusing it on that aspect of neuroscience which concerns the processing, saving and transformation of information. Therefore, we 
would like to have among our contributors especially those authors of papers dealing with the

- theory of neural networks, natural and artificial,

- methods of neurocomputing,

- parallel computing methods,

- synthesis and construction of neurocomputers,

- distributed and parallel computer systems,

- mass-parallel information processing,

- biophysics and neuroscience,

- applications of neurocomputing in science and engineering.

Of course, the selection of good papers is not an easy task and therefore we hope that the International Editorial Board of our Journal, membership in which was accepted by well-known people in neuroscience, will be a great help to us. We are grateful to many of the for their support and encouragement without which we would hardly be able to start this Journal in such considerably short time.

\section{The structure of Neural Network World}

Like some other scientific journals, the profile of which we have known for many years, we would like to offer to our readers not only the presentation of interesting and significant papers, improving the general knowledge of neuroscience, but also some other useful services. Of course, we shall accept for publication in Neural Network World not only the regular Papers of the usual size 20 to 35 pages, but also the short contributions of a few pages' size, which we shall present as Letters (I know very well from my own long experience in circuit and the systems theory, that quite often very important ideas appear just in such short messages).

Besides this, we shall insert in some of our issues a Tutorial section, in which we would like to present to the readers (especially to those who are just starting their interest in this field) some useful tools, like survey views, descriptions of algorithms and information on computer programs.

The almost standard service in any scientific journal consists in the presentation of a Literature survey and Book reviews. However, we think that especially in our field of neuroscience this is of special importance, because of its high dynamics. We shall take a great care with it and try to inform the readers about all the interesting presentations which come in the Scientific Information System of the Institute of Computer and Information Science of the Academy of Sciences in Prague, with which we cooperate in this respect.

We also would like to inform the readers about the most interesting Coming Events in neuroscience, about the New Products appearing in the market and about the Neurocomputer Companies, the number of which is substantially increasing. 


\section{Neural Network World 2/2020}

Such a structure of our Journal is of course not a rigid skeleton. It will be modified according to the experience with respect to the development of the whole field of neuroscience.

\section{Appreciation}

The creation of a new scientific journal in neuroscience is an exciting, but complicated and not easy activity.

The undertakings are large, and I personally feel quite strongly the responsibility to do all the necessary things well. Therefore, I am very indebted to all the people who helped us, above all to the members of the International Editorial Board, to the contributors, to the staff of the IDG Company Czechoslovakia in Prague and to all my colleagues working as Associate Editors. They have done an excellent work without which we could not succeed to publish the first issue of Neural Network World in such tight time schedule.

We hope that the results of this hard work, which we now are giving into your hands, our respectful reader, will be interesting and useful for you and that you will appreciate our efforts.

Mirko Novák Editor-in-Chief 